\title{
Should TIA and Minor Stroke Patients Be Kept Out of The Hospital?
}

Annemarei Ranta, MD, PhD, FRACP ${ }^{1}$, Rachael Hunter, $\mathrm{MSc}^{2}$

${ }^{1}$ Department of Neurology, Wellington Regional Hospital and Department of Medicine, University of Otago, Wellington, New Zealand

${ }^{2}$ Research Department of Primary Care and Population Health, University College London, London, United Kingdom

\section{Correspondence:}

Prof. Anna Ranta, MD, PhD, FRACP

Level G G04

Department of Medicine

School of Medicine and Health Sciences

University of Otago, Wellington

Email: anna.ranta@otago.ac.nz

Word Count: 1014

Key Words: Transient Ischemic attack, Stroke, Health Service Delivery, Inpatient, Outpatient 
As a medical emergency, Transient Ischemic Attack (TIA) requires early, appropriate management to minimize the risk of stroke. ${ }^{1}$ Traditionally, we have admitted all patients with TIA or minor stroke to the hospital presuming that this would offer the best care with the least possible delay. Over the past two decades increasing evidence has emerged demonstrating the feasibility of urgent outpatient TIA management with multiple, alternative models of care proposed, implemented, and evaluated. ${ }^{2}$

A key intervention in $\mathrm{TIA} /$ minor stroke management, whether as an in- or outpatient, is rapid access to stroke specialists. ${ }^{3}$ Most current TIA service models rely on telephone, electronic, or emergency department triage of referrals to decide which patients require specialist review either as an inpatient or an outpatient. ${ }^{2}$ Locales without rapid outpatient clinics managed patients with TIA exclusively in the inpatient setting. ${ }^{4,5}$ Many still presume that universal TIA admission confers greater benefit over outpatient management and the literature offers little on outpatient management of strokes.

In this issue of Neurology Hastrup et al. ${ }^{6}$ describe a same-day outpatient TIA clinic following a telephone call to a neurologist as the primary specialist contact point for all TIA/minor stroke patients referred between $8 \mathrm{am}$ and $6 \mathrm{pm}$. They primarily admitted high-risk patients $(23.5 \%$ in the reported cohort). Risk stratification included the ABCD2 score for TIA and National Institutes of Health Stroke Scale for minor stroke, magnetic resonance imaging, neck vessel imaging, presence of cardioembolic source, and indication for revascularization. Patient evaluations and investigations occur in an ambulatory clinic adjacent to the acute stroke unit (ASU), bypassing the emergency department. Patients with ongoing symptoms have access to on site and early community therapist intervention.

The authors compared the outcome of a cohort of 1,076 patients managed using this approach between 1 September 2013 and 31 August 2014 with matched historic controls (one year prior; same center) for TIA patients and matched historic and contemporary controls (same time period; different center) for minor stroke patients using the Danish National Patient Register.

Of the 1,076 patients referred 215 (20.0\%) had a stroke, 171 (15.9\%) a TIA, 124 (11.5\%) another neurovascular diagnosis, and 566 (52.6\%) a non-neurovascular diagnosis.

Compared with universal ASU admission without prior outpatient assessment, minor stroke patients undergoing the described service model experienced shorter risk-adjusted length of acute hospital stay (median 1 vs 3 days; adjusted length of stay ratio (LOSR) 0.49 (95\% Cl 0.33-0.71)) and shorter risk-adjusted overall length of stay including rehabilitation (median 1 vs 4 days; adjusted LOSR 0.67 (95\% $\mathrm{Cl} 0.46-1.00)$. In addition, 30-day readmission rates were lower (3.2\% vs $11.6 \%$; aHR 0.23 (0.090.59 ) and 'all-or-none' of 10 process of performance measures were higher in patients in the outpatient cohort (84.3\%) versus admission cohort (64.9\%) with risk ratio of 1.30 (1.15-1.47). The groups had similar mortality and delayed readmission. Those with TIA had similar patterns observed.

The authors only captured 7-day stroke recurrence rates for the intervention group. For confirmed TIA and minor stroke patients in the outpatient clinical model group $0.6 \%(1 / 215)$ 'low risk' and $2.2 \%$ $(1 / 167)^{\prime}$ 'high risk' patients experienced stroke recurrence by day seven. They admitted $20.9 \%$ of the low-risk and $73.1 \%$ of high-risk patients to the ASU. 
These results make a strong case for initial evaluation of all TIA/minor stroke patients in a specialist outpatient unit instead of universal admission to a stroke unit. The service model may cost less per patient, free up hospital bed-days for more severe stroke patients, and reduce stroke related readmissions ( $>24$ hours hospital stay after the first admission). Certainly, the lack of difference in mortality and early stroke recurrence rates comparable to other published data suggest no harm from this service model. ${ }^{2}$

These findings suggest that primary outpatient management with only selective hospital admission may in fact be better than universal hospital admission. The authors postulate that more consistent implementation of best practice care in outpatient clinics, evidenced by higher rates of achieving the 10 process of performance measure and a lower rate of contracting hospital acquired infections account for this benefit. A 7-day follow-up by telephone offered to half of the clinic cohort, not standardly used in the hospitalized cohorts, may also have prevented readmissions.

This study had several limitations. Firstly, as an observational study, it must be interpreted with some caution. Secondly, the complicated use of two different control groups might introduce bias. Use of historical data in particular can introduce bias due to temporal trends in care independent of the intervention, for example the introduced 7-day telephone follow-up call highlighted by the authors. Third, their data sources lacked 3-month stroke recurrence rate and thus limits comparison to other studies necessitating reliance on surrogate markers such as readmission. Finally, the proposed model relies on rapid availability of outpatient services potentially unachievable in many settings including same-day therapist assessment and Holter monitors.

However, despite these limitations, this study brings to the fore the need to reassess the value we place on hospital admissions. We know from many studies that early stroke specialist involvement is a key intervention for TIA and minor stroke patients. We now have to question whether admitting all TIA and minor stroke patients to an ASU offers the best opportunity to achieve optimal outcomes or whether rapid access stroke specialist outpatient TIA/minor stroke model with only selective subsequent ASU admission should in fact become the new standard of care. If so, we need to prioritize establishing rapid outpatient pathways including effective pre-clinic triage to reduce unnecessary assessment of stroke mimics, same-day outpatient access to key investigations, early outpatient therapist involvement, and early follow-up at least by telephone. Clinical trial comparison of out- versus inpatient TIA and minor stroke management would form an excellent focus of future research in this area. 


\section{References:}

1. Rothwell PM, Giles MF, Chandratheva A, et al. Effect of urgent treatment of transient ischaemic attack and minor stroke on early recurrent stroke (EXPRESS study): a prospective population-based sequential comparison. Lancet 2007;370:1432-42.

2. Ranta A, Barber PA. Transient ischemic attack service provision: A review of available service models. Neurology 2016.

3. Giles MF, Rothwell PM. Risk of stroke early after transient ischaemic attack: a systematic review and meta-analysis. Lancet Neurol 2007;6:1063-72

4. Nguyen-Huynh MN, Johnston SC. Is hospitalization after TIA cost-effective on the basis of treatment with tPA? Neurology 2005;65:1799-801.

5. Joshi JK, Ouyang B, Prabhakaran S. Should TIA patients be hospitalized or referred to a sameday clinic?: a decision analysis. Neurology 2011;77:2082-8.

6. Hastrup et al. Specialised outpatient clinic vs stroke unit for TIA and minor stroke: a cohort study. Neurology 2020;XX:XX. 